\title{
Exosome microRNA signatures in patients with complex regional pain syndrome undergoing plasma exchange
}

\author{
Sujay Ramanathan', Sabrina R. Douglas ${ }^{1}$, Guillermo M. Alexander², Botros B. Shenoda' ${ }^{1}$, James E. Barrett ${ }^{1,2}$, \\ Enrique Aradillas ${ }^{2,4}$, Ahmet Sacan ${ }^{3}$ and Seena K. Ajit ${ }^{*}$
}

\begin{abstract}
Background: Therapeutic plasma exchange (PE) or plasmapheresis is an extracorporeal procedure employed to treat immunological disorders. Exosomes, nanosized vesicles of endosomal origin, mediate intercellular communication by transferring cargo proteins and nucleic acids and regulate many pathophysiological processes. Exosomal miRNAs are potential biomarkers due to their stability and dysregulation in diseases including complex regional pain syndrome (CRPS), a chronic pain disorder with persistent inflammation. A previous study showed that a subset of CRPS patients responded to PE.

Methods: As a proof-of-concept, we investigated the PE-induced exosomal miRNA changes in six CRPS patients. Plasma cytokine levels were measured by HPLC and correlated with miRNA expression. Luciferase assay following cotransfection of HEK293 cells with target 3'UTR constructs and miRNA mimics was used to evaluate miRNA mediated gene regulation of target mRNA. Transient transfection of THP-1 cells with miRNA mimics followed by estimation of target gene and protein expression was used to validate the findings.

Results: Comparison of miRNAs in exosomes from the serum of three responders and three poor-responders showed that 17 miRNAs differed significantly before and after therapy. Of these, poor responders had lower exosomal hsa-miR-338-5p. We show that miR-338-5p can bind to the interleukin 6 (IL-6) $3^{\prime}$ untranslated region and can regulate IL-6 mRNA and protein levels in vitro. PE resulted in a significant reduction of IL-6 in CRPS patients.

Conclusions: We propose that lower pretreatment levels of miR-338-5p in poor responders are linked to IL-6 levels and inflammation in CRPS. Our data suggests the feasibility of exploring exosomal miRNAs as a strategy in patient stratification for maximizing therapeutic outcome of PE.
\end{abstract}

Keywords: Plasma exchange, Exosomes, miRNA, Inflammation, Biomarker

\section{Background}

Therapeutic plasmapheresis or plasma exchange (PE) is an extracorporeal blood purification technique designed to remove large-molecular-weight substances [1]. For $\mathrm{PE}$ to be a rational choice as a blood purification technique, at least one of the following conditions should be met. The substance to be removed is sufficiently large

\footnotetext{
*Correspondence: ska52@drexel.edu

${ }^{1}$ Pharmacology \& Physiology, Drexel University College of Medicine, 245

North 15th Street, Mail Stop 488, Philadelphia, PA 19102, USA

Full list of author information is available at the end of the article
}

( $\geq 15 \mathrm{kDa}$ ), has a comparatively prolonged half-life so that its removal provides a therapeutically beneficial period of reduced serum concentration, or the rapid removal of acutely toxic substance resistant to conventional therapy is clinically indicated [1]. PE is a widely used therapeutic procedure in many immunologic renal and neurological disorders, enabling the removal of pathogenic antibodies and circulating immune complexes that can cause inflammation. Often used in combination with immunosuppressive therapies, PE has led to a steady increase in survival rates over the last 40 years in these diseases with a poor prognosis without treatment [2]. 
Complex regional pain syndrome (CRPS) is a chronic neuropathic pain disorder and the patient population is heterogeneous with symptoms encompassing sensory, motor and autonomic dysfunction. This disabling disease is characterized by allodynia (pain from a non-painful stimulus), hyperalgesia (heightened sensitivity to pain), changes in skin color, temperature, edema, alterations in hair, skin or nail, dystonia, and tremors [3, 4]. The pathophysiological mechanisms of CRPS are not fully understood, although a moderate association has been observed with autoantibodies against adrenoceptors [5]. PE can remove proinflammatory cytokines, immune complexes and autoantibodies to adrenoceptors, and thus relieve localized and systemic symptoms [6-9]. A recent study reported that $\mathrm{PE}$ was effective on a subset of CRPS patients with reduced epidermal nerve fiber density count, consistent with a small fiber neuropathy, with 30 out of 33 (91\%) patients reporting pain relief [10].

In addition to high molecular weight entities, plasma also contains extracellular vesicles including the 30-150 nm exosomes. These circulating exosomes are secreted by a variety of cell types and transport various cargo molecules including miRNA, long noncoding RNA, mRNA, lipids, DNA and proteins. The release and uptake of exosomes play an important role in intercellular communication [11]. The content of exosomes is regulated under homeostasis, and often a dysregulation precedes or ensues pathology [12, 13]. Distinct expression patterns of circulating miRNAs have been associated with a number of diseases [14]. Widely recognized for their role as fine tuners of gene expression, miRNAs that mediate posttranscriptional regulation influence virtually all aspects of cellular processes and are also transported via exosomes $[11,15,16]$. These small noncoding RNAs regulate gene expression by binding predominantly to the $3^{\prime}$ untranslated region ( $3^{\prime} \mathrm{UTR}$ ) of mRNAs by 6 - to 8-basepair seed sequence complementarity. Upon binding, miRNAs can induce mRNA degradation or translational repression and thus negatively regulate the expression of target genes $[15,16]$.

A recent study investigated whether the removal or reduction of circulating miRNAs with plasma exchange improved pathologies caused by miRNAs [17]. The study evaluated miRNAs in three patients with systemic lupus erythematosus undergoing plasmapheresis and showed that a large number of circulating miRNAs in plasma were separated with apheresis [17]. Our previous study has shown that a larger number of circulating miRNAs are differentially expressed (or packaged) into exosomes in CRPS patients compared to exosomes from control subjects [18]. To the best of our knowledge, there are no studies to date investigating exosome composition in serum before and after PE. Thus, we sought to determine the feasibility of using circulating miRNAs transported within exosomes in serum samples of CRPS patients before and after PE as a potential molecular signature or biomarker, by performing a proof-of-concept study in three patients who responded and three patients who did not respond to PE.

\section{Methods \\ Standard protocol approvals, registrations, and patient consents}

This is a retrospective case series study of CRPS patients seen at the Drexel University College of Medicine pain clinic that met the Budapest consensus criteria for CRPS and received PE as treatment for their illness between September 2012 and June 2014. All participants agreed to provide blood samples for this study after giving informed consent as approved by the institutional review board.

\section{Plasma exchange and patient evaluation}

The patients were asked to provide blood samples before and after PE. Patients with autoimmune or immunodeficiency conditions were excluded. Patient records were reviewed from which data regarding demographics, CRPS signs and symptoms, duration of illness and response to PE were obtained. PE was performed in patients with refractory CRPS over 2 weeks at the Hahnemann University Hospital, Philadelphia. All patients had full cardiac and neuropsychological clearance before PE. Patients were asked to rate their overall pain before, during, and after the apheresis using a 11-point numerical rating scale (NRS) from 0 (no pain) to 10 (the worst pain imaginable).

\section{Characterization of exosomes by nanoparticle tracking analysis, electron microscopy and western blot analysis} One milliliter of serum from CRPS patients was diluted in equal volume of PBS and centrifuged at $2000 \times g$ for $30 \mathrm{~min}$ at $4{ }^{\circ} \mathrm{C}$. The supernatant was diluted to final volume of $24 \mathrm{ml}$ in $1 \times \mathrm{PBS}$ and centrifuged at $12,000 \times g$ for $45 \mathrm{~min}$ at $4{ }^{\circ} \mathrm{C}$. The supernatant was filtered through a $0.22 \mu$ filter and centrifuged at $110,000 \times g$ for $70 \mathrm{~min}$ at $4{ }^{\circ} \mathrm{C}$. The exosome pellet obtained was washed in $25 \mathrm{ml}$ $1 \times$ PBS without ions and centrifuged at $110,000 \times g$ for $70 \mathrm{~min}$ at $4{ }^{\circ} \mathrm{C}$. The exosome pellet was resuspended in $100 \mu \mathrm{l}$ of PBS for use in nanoparticle tracking analysis, electron microscopy and protein estimation.

\section{Nanoparticle tracking analysis}

Exosomes in PBS were analyzed for size and concentration using the NanoSight NS300 according to the manufacturer's protocol (Malvern Instruments, MA, USA). Samples were diluted to $\sim 10^{7}-10^{9}$ particles $/ \mathrm{ml}$ and 
continuously injected with a syringe pump and three videos (30 s each) were captured for particle analysis. Nanoparticle tracking analysis was performed using NTA 3.2 software.

\section{Electron microscopy}

Ten microliters of PBS resuspended exosomes were coated on Ni-formvar grids and incubated for $20 \mathrm{~min}$ at RT. The grids were washed on $50 \mu \mathrm{l}$ drops of $0.1 \mathrm{M}$ Sorensen's phosphate buffer $(\mathrm{pH}$ 7.2) for $5 \mathrm{~s}$ each for a total of five times. The grids were blot dried perpendicularly on whatman \#1 filter paper. Negative staining and embedding were performed by incubating the grids on $0.5 \%$ uranyl acetate (in a $0.2 \%$ methyl cellulose solution) for $10 \mathrm{~min}$ at $4{ }^{\circ} \mathrm{C}$. The excess solution was blotted on a Whatman paper, air dried and imaged in a JEOL Transmission Electron Microscope (JEM 1230). Alternatively, the exosomes were immunolabelled for the exosome marker CD81 and crosslinked with $1 \%$ glutaraldehyde, and probed with $6 \mathrm{~nm}$ gold secondary antibody, followed by negative staining and embedding.

\section{Western blotting}

Protein concentration was estimated using a DC Protein assay (Bio-Rad Laboratories, CA, USA); $5 \mu \mathrm{g}$ of exosomes isolated from CRPS serum were resolved on a reducing 12\% SDS-PAGE, transferred to PVDF membrane and blocked in Odyssey Blocking buffer (927-50100, LI-COR Biosciences) for $2 \mathrm{~h}$. The membrane was incubated in rabbit anti-CD63 antibody (ab68418, Abcam) overnight at $4{ }^{\circ} \mathrm{C}$, washed thrice in TBST, $10 \mathrm{~min}$ each, and incubated in goat anti-rabbit 680RD IgG (925-68071, LI-COR Biosciences) for $45 \mathrm{~min}$ at RT, washed thrice in TBST for 10 min each, and imaged on an Odyssey Fc imaging system.

\section{Exosome miRNA profiling}

RNA was isolated from exosomes using a miRvana miRNA isolation kit (Life technologies) following the manufacturer's protocol. Taqman low-density array microfluidic cards version A and B (Applied Biosystems, Foster City, CA) were used to profile miRNAs in $100 \mathrm{ng}$ of total RNA as previously described [19]. miRNA species with $C T$ values 35 and higher were treated as undetected. Fold change was calculated from raw CT values using the $2^{-\Delta \Delta C T}$ method [20]. The mean CT values of the 10 miRNAs with the lowest standard deviations across all samples were used as the endogenous control in the calculation of $\Delta C T$. Statistical significance of differences in $\Delta \mathrm{CT}$ values was calculated by a two-tailed paired t-test for comparison of pre- and post-PE samples and by a two-tailed independent $\mathrm{t}$-test for comparison of other experimental groups. A p-value threshold of 0.05 and a fold-change of 2 were used to select significant differentially expressed miRNAs between experimental groups.

\section{Cell culture}

HEK293 cells obtained from the American Type Culture Collection (ATCC) was maintained in Dulbecco's Modified Eagle's Medium (DMEM) supplemented with 10\% fetal bovine serum at $37{ }^{\circ} \mathrm{C}$ in $5 \% \mathrm{CO}_{2}$. Human monocytic THP-1 cells (TIB-202, ATCC) cells were maintained in RPMI-1640 medium containing $10 \%$ fetal bovine serum (FBS).

\section{Luciferase reporter assay}

The $3^{\prime}$ UTR luciferase reporter constructs for IL-6 (NM_000600.2) was purchased from GeneCopoeia. The miRNA mimics for human miR-338-5p (MC12825), anti-miR-338 (MH12825) and scrambled negative control (4464058) was purchased from Life technologies. HEK293 cells were co-transfected with mir-338-5p, anti-miR-338 or miRNA scrambled control and luciferase reporter plasmid containing the $3^{\prime} \mathrm{UTR}$ of human IL-6 using Lipofectamine LTX (Life Technologies, Carlsbad, CA) for $48 \mathrm{~h}$. The Luc-Pair miR Luciferase assay kit (GeneCopoeia) was used to measure firefly and Renilla luciferase activity according to the manufacturer's instruction. Firefly luciferase measurements normalized to Renilla luciferase was used as a transfection control. The data expressed as percentage of control is the average of three independent experiments.

\section{miR-338-5p overexpression in THP-1 cells}

Transfections were performed following the manufacturer's protocol for RNAiMax transfection reagent using either miR-338-5p or negative control with the following modifications. For each well of a 6 -well plate, $7.5 \mu \mathrm{l}$ RNAiMax reagent was diluted in $150 \mu$ of serum-free media, and $30 \mathrm{pmol}$ of miR-338-5p or control mimic was diluted in $150 \mu \mathrm{l}$ of serum-free media individually. The dilutions were combined and incubated at room temperature for $15 \mathrm{~min}$. This transfection complex $(300 \mu \mathrm{l})$ was added to $0.5 \times 10^{6}$ cells/well in $1.7 \mathrm{ml}$ serum containing media in 6-well plates and incubated for $6 \mathrm{~h}$ at $37^{\circ} \mathrm{C}$, after which the media was changed. After $24 \mathrm{~h}$, cells were treated with $1 \mu \mathrm{g} / \mathrm{ml}$ lipopolysaccharide (LPS) in complete culture media for $6 \mathrm{~h}$. Exosome deplete media was used in all experiments. Cells were collected by centrifugation at $135 \times g$ for $5 \mathrm{~min}$ at $4{ }^{\circ} \mathrm{C}$ and the conditioned media was stored at $4{ }^{\circ} \mathrm{C}$. The cell pellet was washed with $1 \times$ PBS and resuspended in either RNA lysis buffer (mirVana kit; Life Technologies) containing $0.5 \mathrm{U} / \mu \mathrm{l}$ RNAsin Plus (Promega; Madison, WI) for RNA isolation or $1 \times$ radioimmunoprecipitation assay (RIPA) buffer 
containing protease inhibitor cocktail (Thermo Scientific; Waltham, MA) for protein analysis.

\section{CDNA synthesis and qPCR for mRNAs}

mRNA was isolated using the miRVana kit (Life technologies). The Maxima cDNA synthesis kit (Thermo Scientific) was used to generate cDNA and $2 \mu \mathrm{l}$ cDNA was used for Taqman based quantitative real time mRNA analysis containing $10 \mu \mathrm{l}$ Taqman Fast Universal polymerase chain reaction (PCR) master mix $(2 \times)$ no AmpErase UNG (Life Technologies), $1 \mu \mathrm{l}$ Taqman primer-probe $(20 \times)$, in up to $20 \mu \mathrm{l}$ nuclease-free water. GAPDH was used as the normalizer and one-way ANOVA was used to perform statistical analysis. Assay IDs were as follows: Hs00985639_m1 [IL-6], 4325792 (GAPDH) (Applied Biosystems, Carlsbad, CA).

\section{Enzyme-linked immunosorbent assay (ELISA) for IL- 6 in cell culture media}

Supernatants collected after miR-338-5p or control miRNA transfections in THP-1 cells were used to perform ELISA for secreted IL-6 using the human IL-6 quantikine ELISA kit (D6050) according to the manufacturer's protocol (R\&D Systems; Minneapolis, MN).

\section{Cytokine measurement in patient plasma}

For plasma isolation, blood was collected into EDTAcoated (purple top) vacutainers. The plasma was separated by centrifugation $\left(3000 \times g\right.$ for $15 \mathrm{~min}$ at $\left.4{ }^{\circ} \mathrm{C}\right)$, split into $250 \mu \mathrm{l}$ aliquots and stored at $-70{ }^{\circ} \mathrm{C}$ until assayed. The MILLIPLEX MAP Human High Sensitivity T Cell Panel, HSTCMAG-28SK (Millipore, Billerica, MA) was used to determine plasma levels of 14 cytokines. The minimum detectable concentration for IL- 6 and TNF- $\alpha$ was 0.11 and $0.16 \mathrm{pg} / \mathrm{ml}$ respectively. All assays were performed in duplicate according to the manufacturers' instructions. Assay results were determined on a Luminex-200 (Luminex, Austin, TX).

\section{Statistical analysis}

Data are presented as mean \pm the standard error of the mean from three or more independent experiments. Student $t$-test was used for determining the statistical significance. Treatment effects were analyzed with a one-way analysis of variance (ANOVA). Pairwise comparisons between means were tested using the post hoc Dunnet method. Error probabilities of $p<0.05$ were considered statistically significant. Paired t-test with Bonferroni correction for multiple assays was used for analysis of the cytokine panel. Error probabilities of $p<0.00357$ were considered statistically significant.

\section{Results}

Isolation and characterization of exosomes

Exosomes were isolated from the serum obtained from six CRPS patients, grouped retrospectively as three responders and three non-responders both before and after PE (Fig. 1, Table 1). The exosomes displayed a size below $100 \mathrm{~nm}$ under a transmission electron microscope (TEM) (Fig. 2a, b). Exosomes are characterized by the presence or enrichment of endosome-derived membrane proteins such as tetraspanins (CD9.CD63, CD81), flotillins, Alix and Tsg101. The presence of such characteristic exosomal proteins was also assessed using immunogold labeling technique for CD81 by TEM (Fig. 2c) and western blotting for CD63 (Fig. 2d). Nanoparticle tracking analysis of serum exosomes indicated particles with a mean diameter of $85.7 \pm 0.9 \mathrm{~nm}$ and a concentration of $3.94 \times 10^{11} \pm 1.6 \times 10^{10}$ particles $/ \mathrm{ml}$ (Fig. 2e). The metrics of exosome isolation, purity and characterization was submitted on EV track [21] and the EV-metric score is $44 \%$.

\section{Differentially expressed miRNAs between responders and poor responders of $\mathrm{PE}$}

Exosomal miRNAs isolated from six CRPS patients, before and after PE, were used to profile a total of 754 miRNAs. The mean CT values of the 10 miRNAs with the lowest standard deviations across all samples were used as the endogenous control in the calculation of $\Delta \mathrm{CT}$ excluding miRNAs with a $\mathrm{CT}$ value of $>35$ in any sample. Statistical significance of differences in $\triangle \mathrm{CT}$ values was calculated by a two-tailed paired $\mathrm{t}$-test for comparison of pre- and post-PE samples and by a two-tailed unpaired t-test for comparison of other experimental groups. A p-value threshold of 0.05 and a fold-change of 2 were used to select significantly differentially expressed miRNAs between experimental groups (Table 2, Fig. 3, Additional file 1: Table S1). The comparison between two

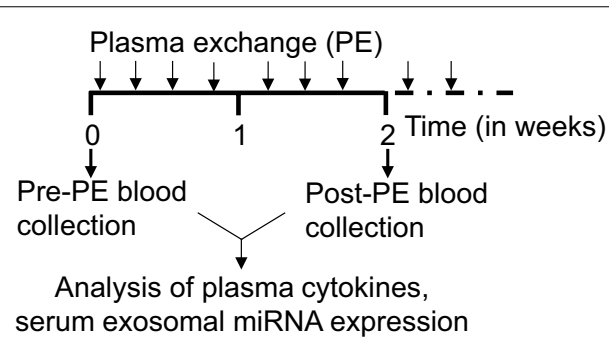

Fig. 1 Schematic of plasma exchange (PE) protocol and sample collection for downstream analysis. Blood was collected from CRPS patients before PE and following a series of five to seven more PE distributed over 2 weeks. Plasma was used to assay the cytokines, while serum was used for exosome isolation and downstream miRNA profiling 


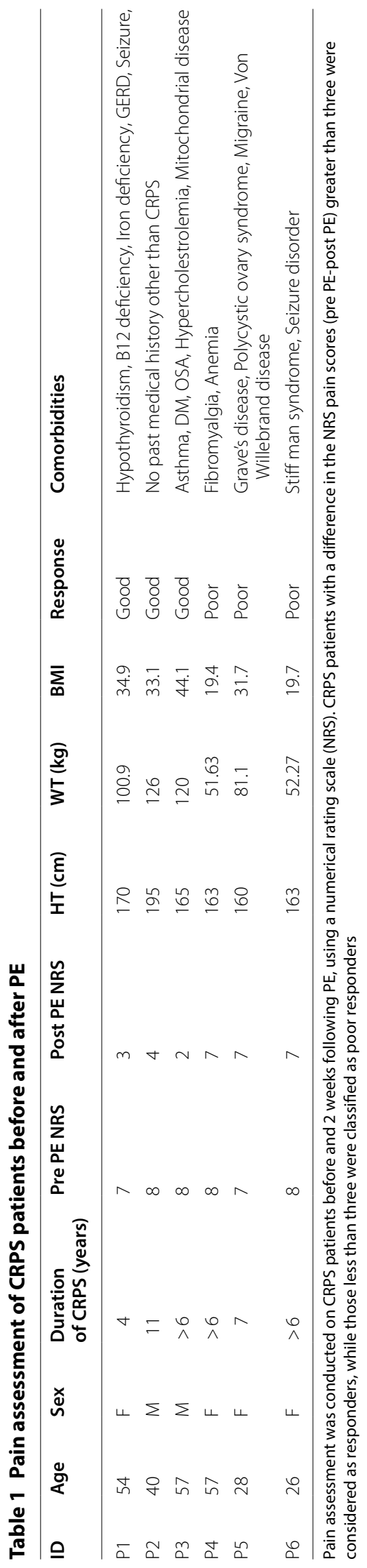



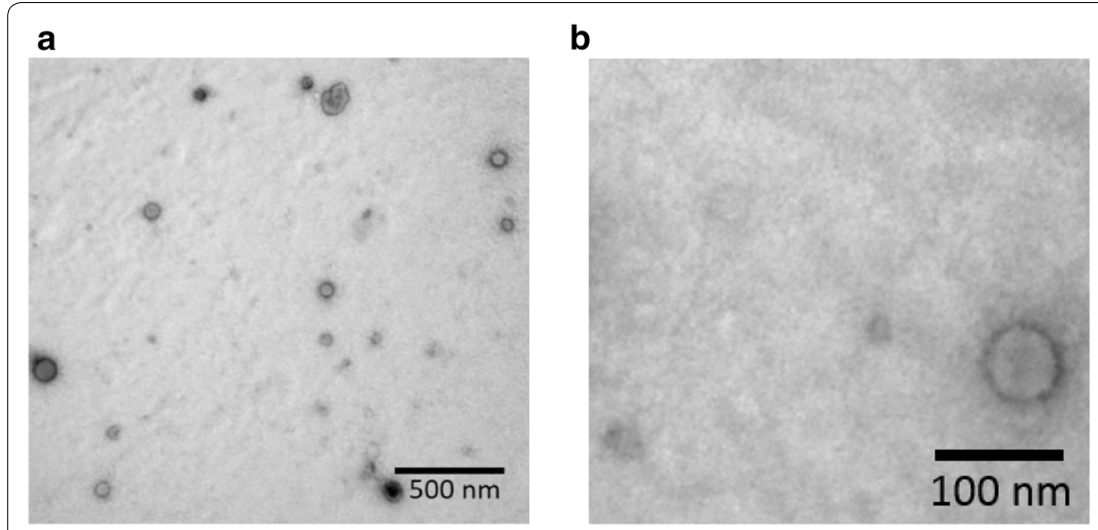

C
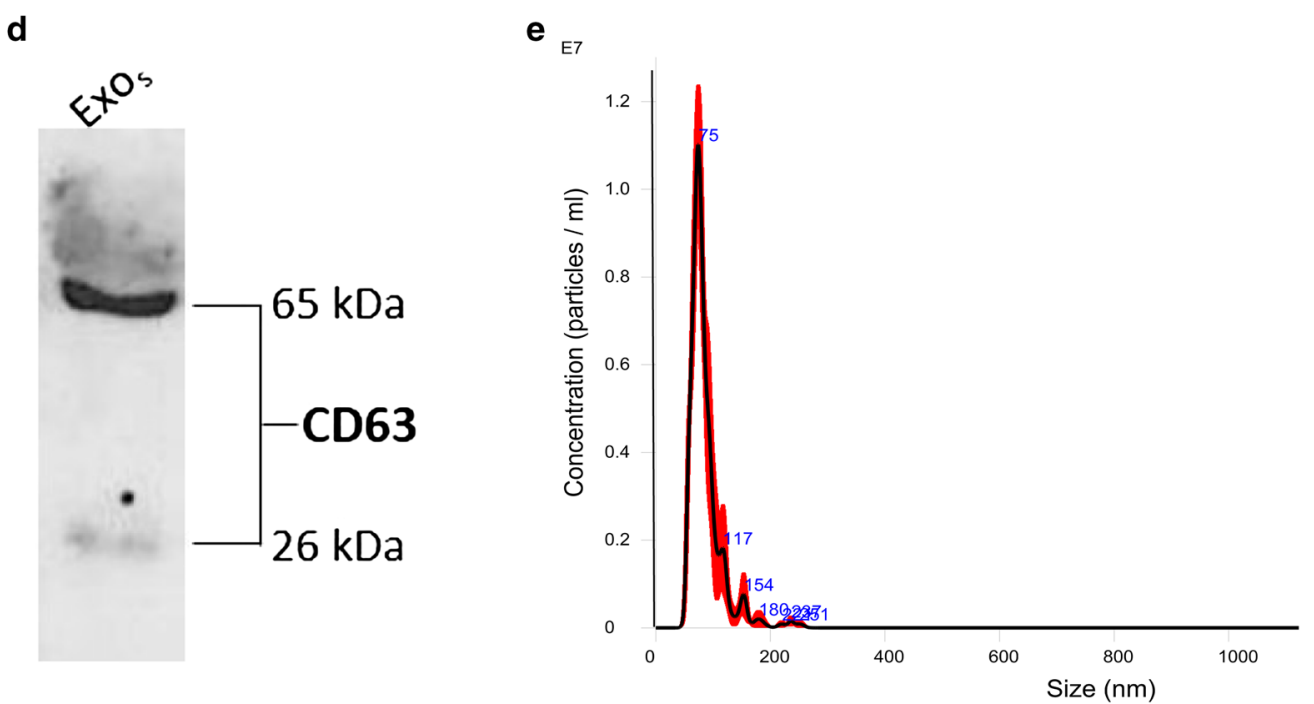

Fig. 2 Characterization of exosomes purified from the serum of CRPS patients. Exosomes were isolated from the serum of CRPS patients using differential ultracentrifugation. The exosomes were observed by TEM under a Toshiba H8000 or JEM1 230 microscope. Exosomes were fixed and negatively stained with uranyl acetate for morphology $(\mathbf{a}, \mathbf{b})$ or immunolabelled for the exosome marker CD81 and probed with $6 \mathrm{~nm}$ gold secondary antibody, following negative staining (c). Exosome proteins isolated from PE patient serum (Exos) were resolved on a reducing $12 \%$ SDS-PAGE and western blotting was used to confirm the presence of exosome marker protein CD63 (d). Nanoparticle tracking analysis of serum exosomes indicated particles with a mean diameter of $85.7 \pm 0.9 \mathrm{~nm}$ and a particle concentration of $3.94 \times 10^{11} \pm 1.6 \times 10^{10} \mathrm{particles} / \mathrm{ml}(\mathbf{e})$

groups is depicted as $\log 2$ fold change values. A positive number in the fold change for $\mathrm{nb} / \mathrm{rb}$, it means $\mathrm{nb}$ is higher than $\mathrm{rb}$. A negative fold change number for $\mathrm{nb} /$ $\mathrm{rb}$ comparison means $\mathrm{nb}$ is lower. To investigate differential miRNA expression between responders and nonresponders prior to $\mathrm{PE}$, we analyzed the miRNAs from the pretreatment groups (column 2 of Table 2, Fig. 3). Of the nine miRNAs that were significantly different between responders and non-responders prior to treatment, two were upregulated and seven downregulated in non-responders relative to responders. This suggests that the exosomal molecular signature differed even prior to PE and could be explored further for biomarker potential in predicting treatment response. To determine which miRNAs changed in responders after treatment, miRNA profiles before and after treatment were compared (Table 2, column 3). Four miRNAs changed significantly in responders after treatment with two miRNAs upregulated and two downregulated (Fig. 3). Two miRNAs were altered after PE in non-responders; however, these were different from the miRNA changes observed in responders. To determine differences in miRNA alterations by PE between responders and non-responders, we compared the changes in miRNA levels of each patient. Results from this meta-analysis (Table 2, column 5) shows nine miRNAs that have different degrees of change in responders and poor responders. For example, hsa-let-7a is downregulated in both responders and 
Table 2 Relative expression of exosomal miRNAs in CRPS patients undergoing plasma exchange (PE)

\begin{tabular}{|l|c|c|c|c|c|c|c|c|}
\hline \multirow{2}{*}{ miRnA } & \multicolumn{2}{|c}{$\mathrm{nb} / \mathrm{rb}$} & \multicolumn{2}{c|}{$\mathrm{ra} / \mathrm{rb}$} & \multicolumn{2}{c|}{$\mathrm{na} / \mathrm{nb}$} & \multicolumn{2}{c|}{$\begin{array}{c}\text { meta:na*- } \\
\text { nb/meta:ra*-rb }\end{array}$} \\
\cline { 2 - 10 } & $\mathrm{p}$ & log2fc & $\mathrm{p}$ & log2fc & $\mathrm{p}$ & log2fc & $\mathrm{p}$ & log2fc \\
\hline hsa-let-7a & $4.9 \mathrm{E}-1$ & -2.9 & $8.4 \mathrm{E}-2$ & -4.7 & $5.3 \mathrm{E}-1$ & -0.2 & $3.9 \mathrm{E}-2$ & 4.5 \\
\hline hsa-miR-19b & $4.4 \mathrm{E}-1$ & -1.1 & $9.8 \mathrm{E}-1$ & 0.0 & $1.7 \mathrm{E}-2$ & 1.0 & $2.9 \mathrm{E}-1$ & 1.1 \\
\hline hsa-miR-29b & $9.1 \mathrm{E}-2$ & -5.8 & $8.7 \mathrm{E}-2$ & -6.2 & $6.3 \mathrm{E}-1$ & 0.7 & $4.0 \mathrm{E}-2$ & 6.9 \\
\hline hsa-miR-191 & $2.0 \mathrm{E}-2$ & 1.9 & $4.3 \mathrm{E}-1$ & 1.0 & $6.3 \mathrm{E}-1$ & -0.4 & $3.2 \mathrm{E}-1$ & -1.4 \\
\hline hsa-miR-195 & $5.1 \mathrm{E}-1$ & -1.1 & $6.1 \mathrm{E}-2$ & -2.4 & $1.3 \mathrm{E}-1$ & 1.7 & $1.1 \mathrm{E}-2$ & 4.1 \\
\hline hsa-miR-484 & $4.2 \mathrm{E}-2$ & 1.2 & $6.2 \mathrm{E}-2$ & 1.5 & $4.2 \mathrm{E}-1$ & -0.4 & $2.6 \mathrm{E}-2$ & -1.8 \\
\hline hsa-miR-518b & $2.2 \mathrm{E}-3$ & -7.3 & $4.5 \mathrm{E}-3$ & -6.9 & $4.9 \mathrm{E}-1$ & -0.2 & $2.6 \mathrm{E}-4$ & 6.6 \\
\hline $\begin{array}{l}\text { hsa-miR-542- } \\
\text { 3p }\end{array}$ & $4.7 \mathrm{E}-2$ & -7.9 & $8.5 \mathrm{E}-2$ & -6.4 & $6.0 \mathrm{E}-1$ & 0.8 & $3.9 \mathrm{E}-2$ & 7.3 \\
\hline hsa-miR-618 & $3.9 \mathrm{E}-2$ & -9.8 & $1.5 \mathrm{E}-1$ & -7.0 & $2.9 \mathrm{E}-1$ & -1.7 & $1.8 \mathrm{E}-1$ & 5.3 \\
\hline $\begin{array}{l}\text { hsa-miR-30a- } \\
\text { 3p }\end{array}$ & $7.9 \mathrm{E}-1$ & 1.0 & $2.4 \mathrm{E}-3$ & 3.1 & $2.3 \mathrm{E}-1$ & 2.3 & $5.7 \mathrm{E}-1$ & -0.8 \\
\hline hsa-miR-126\# & $2.9 \mathrm{E}-2$ & -4.7 & $5.8 \mathrm{E}-1$ & -0.5 & $1.1 \mathrm{E}-1$ & 2.4 & $6.3 \mathrm{E}-2$ & 2.9 \\
\hline hsa-miR-34b & $8.5 \mathrm{E}-2$ & -5.9 & $5.3 \mathrm{E}-1$ & 0.3 & $5.1 \mathrm{E}-2$ & 3.1 & $3.0 \mathrm{E}-2$ & 2.8 \\
\hline hsa-miR-551b\# & $4.5 \mathrm{E}-1$ & 1.9 & $1.0 \mathrm{E}-1$ & 5.5 & $2.7 \mathrm{E}-2$ & -6.1 & $5.8 \mathrm{E}-3$ & -11.6 \\
\hline $\begin{array}{l}\text { hsa-miR-19b- } \\
\text { 1\# }\end{array}$ & $2.0 \mathrm{E}-4$ & -15.5 & $1.6 \mathrm{E}-1$ & -3.8 & $5.6 \mathrm{E}-1$ & 3.9 & $2.6 \mathrm{E}-1$ & 7.7 \\
\hline $\begin{array}{l}\text { hsa-miR-338- } \\
\text { 5P }\end{array}$ & $1.2 \mathrm{E}-3$ & -10.5 & $2.1 \mathrm{E}-2$ & -3.0 & $3.3 \mathrm{E}-1$ & 4.5 & $1.0 \mathrm{E}-1$ & 7.5 \\
\hline hsa-miR-1183 & $7.6 \mathrm{E}-1$ & 0.6 & $3.0 \mathrm{E}-2$ & 3.4 & $2.7 \mathrm{E}-1$ & -2.6 & $3.1 \mathrm{E}-2$ & -6.0 \\
\hline hsa-miR-1274B & $4.4 \mathrm{E}-2$ & -1.7 & $3.2 \mathrm{E}-1$ & -1.1 & $9.3 \mathrm{E}-1$ & 0.1 & $3.9 \mathrm{E}-1$ & 1.2 \\
\hline
\end{tabular}

Relative expression of exosomal miRNAs in six CRPS patients pre and post-PE were determined using a Taqman low density array. The significance was determined using two-tailed paired t-test for comparison of pre- and post-plasma exchange samples and by a two-tailed independent t-test for comparison of other experimental groups. A p-value threshold of 0.05 and a fold-change of 2 were used to select significantly differentially expressed miRNAs between experimental groups

Log2fc: Log 2 fold change, orange red: downregulation, green: upregulation, Rb: responders before PE, Nb: non-responders before PE, Ra: responders after PE, Na: non-responders after $\mathrm{PE}, \mathrm{Na}-\mathrm{Nb} / \mathrm{Ra}-\mathrm{Rb}$ (miRNA changes in non-responders over responders as a result of $\mathrm{PE}$ )

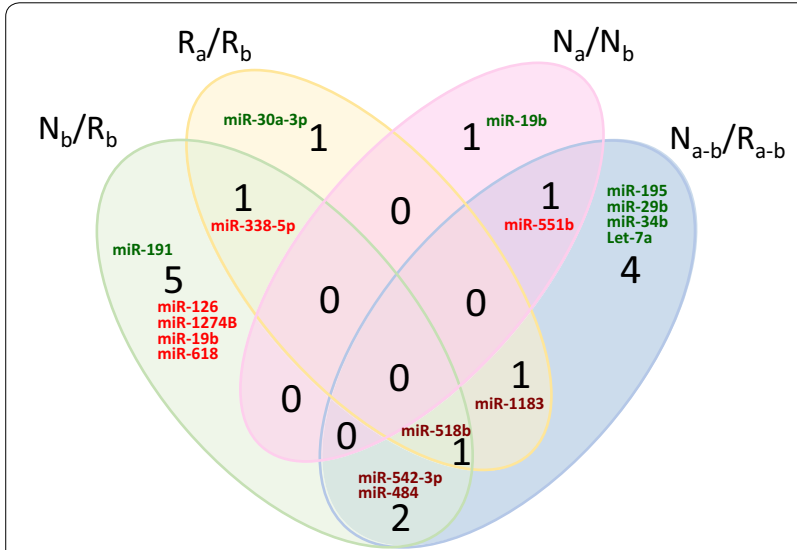

Fig. 3 Exosomal miRNA expression in CRPS patients undergoing plasma exchange. A venn diagram showing relative expression of various miRNAs evaluated from serum exosomes of six CRPS patients before PE and 2 weeks following it. Comparison of miRNA levels are presented in responders $(\mathrm{R})$ and poor responders $(\mathrm{N})$ before (b) and after (a) PE. Upregulated miRNAs are shown in green and downregulated miRNAs in red, while miRNAs that are common between the compared groups but differing in directionality are in dark red. The significance was determined using student t-test $(p<0.05)$ non-responders, but the degree of change in responders is 4.5 -fold greater than in non-responders.

In order to ascertain the potential function of these miRNAs, we employed a combination of bioinformatic algorithms Targetscan and miRDB that predict miRNA targets based on the ability of the miRNA sequence to undergo specific base-pairing within the putative $3^{\prime} \mathrm{UTR}$ in the mRNA target $[22,23]$. Among the pro-inflammatory targets, IL- 6 was among the top candidates for both $\mathrm{miR}-19 \mathrm{a} / \mathrm{b}$ and $\mathrm{miR}-338-5 \mathrm{p}[22,24]$. The regulatory role of miR-19 on IL- 6 has been demonstrated and shown to be indirectly mediated by the binding of miR-19a and miR-19b to Toll-like receptor 2 [25]. The role of miR338-5p in inflammation or CRPS however has not yet been explored. This led us to investigate the biological role of miR-338-5p in regulating inflammatory responses.

\section{Confirmation of miR-338-5p binding to the $3^{\prime}$ UTR of predicted targets}

The predicted interaction of miR-338-5p and IL-6 mRNA (Fig. 4a) was studied using a luciferase reporter assay. The 3'UTR of human IL-6 harboring miR-338-5p 


\section{a}

\section{Seed location in IL6 3'UTR: 363-369 nt}

\section{IL6 3'UTR: 5'-TTTAAAGAAATATTT A TATTGTA-3' miR-338-5p: 3'-GUGAGUCGUGGUCCUAUAACAA-5'}

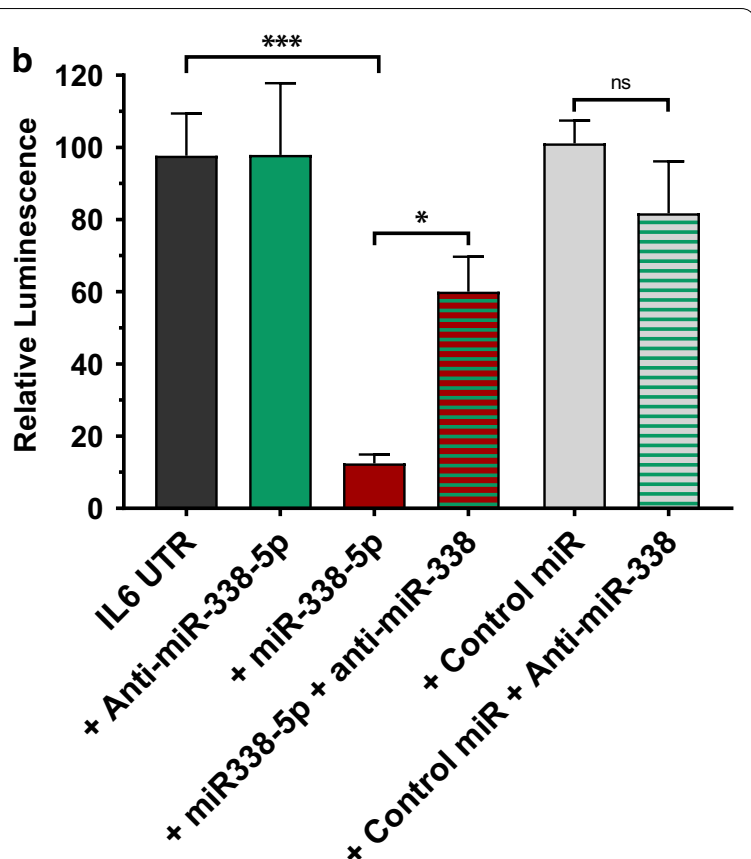

Fig. 4 Luciferase assay to determine miR-338-5p binding to the $3^{\prime} U T R$ of predicted mRNA targets. a The miR-338-5p targeting sequence in the 3'-untranslated region of human IL6 mRNA. b Plasmids with the 3'UTR of IL-6 cloned downstream of the luciferase open-reading frame were cotransfected with miR-338-5p, anti-miR-338 or control miRNA mimic in HEK293 cells. Luciferase activity was measured 48 h after transfection, and the data expressed as percentage of luminescence. Firefly luciferase measurements were normalized to Renilla and the average of three independent experiments is shown. Statistically significant difference from control was calculated using Student t-test, ${ }^{*} p<0.05$ binding sites cloned downstream of the luciferase openreading frame was used to assess miRNA-mRNA binding. HEK293 cells were transiently transfected with plasmids encoding the reporter $3^{\prime} \mathrm{UTR}$ construct and either miR-338-5p mimic, anti-miR-338 or a miRNA control. Firefly luciferase measurements were normalized to Renilla luciferase as a transfection control. A significant reduction was observed in luciferase activity $48 \mathrm{~h}$ after transfection with miR-338-5p, $(\mathrm{p}<0.05)$ confirming the binding of miR-338-5p to the $3^{\prime}$ UTR of IL- 6 mRNA (Fig. 4b). Anti-miRs are complementary to the target miRNAs and used to study loss of function effects of specific miRNAs. Transfection with anti-miR-338 individually or in combination with control miRNA did not alter relative luminescence but when combined with miR338-5p, significantly reversed the inhibition indicating downregulation of miRNA activity.

\section{Transcriptional and translational regulation of IL-6 mRNA by miR-338-5p}

We next assessed the ability of miR-338-5p to modulate levels of the pro-inflammatory IL- 6 mRNA in vitro. IL-6 mRNA and protein levels are usually very low in normal/ unstimulated immune cells. Hence, to assess the observable effects of miR-338-5p on IL-6 mRNA, we stimulated human monocytic leukemia (THP-1) cells with bacterial lipopolysaccharide (LPS). THP-1 cells were transfected with either miR-338-5p mimic or control miRNA for $18 \mathrm{~h}$ and then stimulated with LPS $(1 \mu \mathrm{g} / \mathrm{ml})$ for $6 \mathrm{~h}$, following which endogenous levels of target mRNAs were measured by qPCR. There was a significant reduction in IL-6 mRNA upon miR-338-5p transfection compared to control miRNA transfection, suggesting that miR-338-5p regulates IL-6 via mRNA degradation (Fig. 5a). Conditioned media from THP-1 cells transfected with miR338-5p or control and stimulated with LPS were analyzed by ELISA for IL-6 protein levels. The overexpression of miR-338-5p significantly reduced protein levels of IL-6 secreted into the media (Fig. 5b). Thus, over expression of miR-338-5p resulted in downregulation of both mRNA and protein levels of IL-6.

\section{Plasma levels of cytokines in CRPS patients}

Persistent inflammation is a hallmark of CRPS and an upregulation of proinflammatory cytokines has been demonstrated by multiple approaches in various biofluids including plasma, serum, blister fluid and cerebrospinal fluid [26]. We investigated a panel of 14 cytokines (Additional file 2: Table S2) of which only IL-6 was significantly different following PE. As both miR-19 and miR-338-5p 

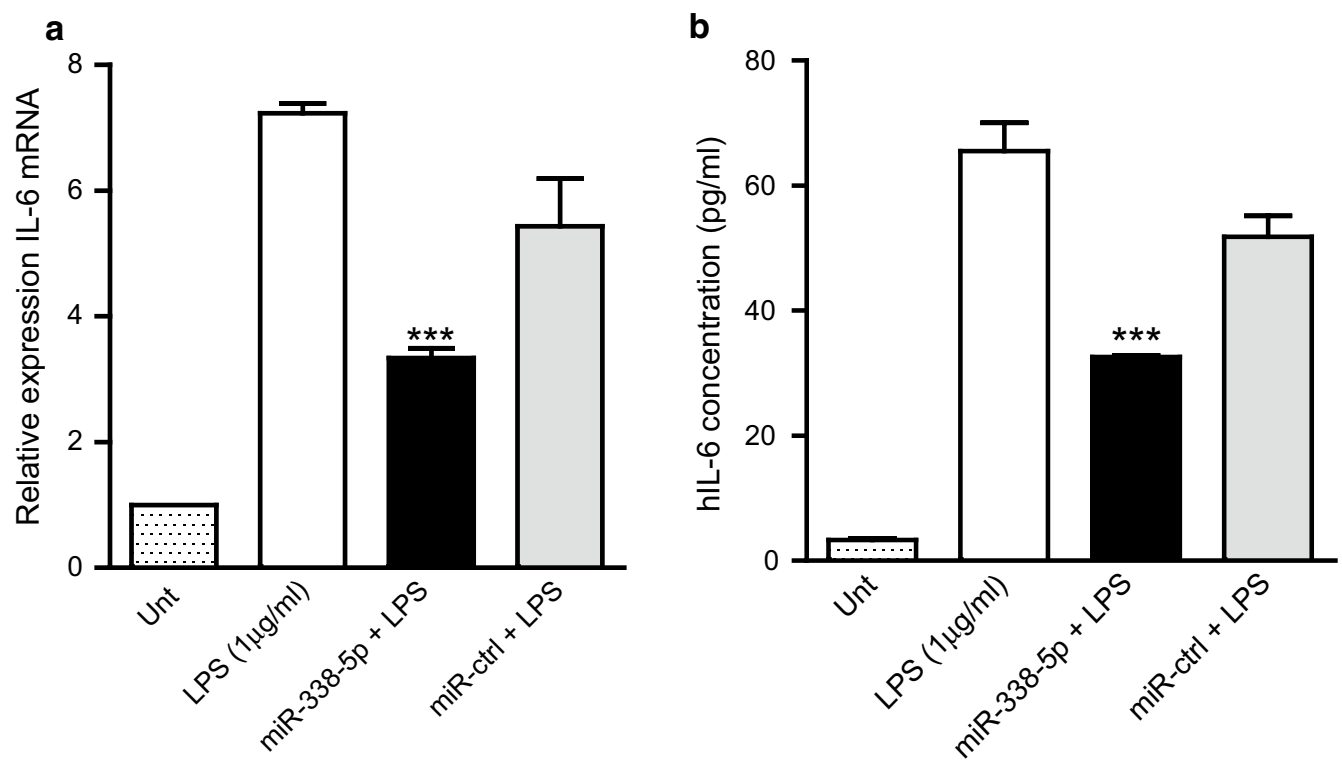

Fig. 5 Expression levels of IL-6 mRNA and protein in THP-1 cells transfected with miR-338-5p followed by $6 \mathrm{~h}$ of stimulation with LPS. a Taqman analysis of endogenous levels of IL-6 after LPS induction, showed that transfection with miR-338-5p reduced IL-6 transcripts compared to control miRNA (miR-ctrl). b Overexpression of miR-338-5p decreased the secreted IL-6 content. ELISA using cell culture supernatants of THP-1 cells stimulated with LPS showed lower levels of the proinflammatory mediator secreted by miR-338-5p transfected cells compared to control miRNA transfection. Significance was determined by one-way ANOVA with Dunnet's post hoc test, ${ }^{* * *} p<0.001$

can target IL-6, we investigated IL-6 in a plasma sample repository available from 18 patients. A significant reduction was observed in the plasma levels of IL- 6 following PE $(n=18)$, while TNF $\alpha$ levels did not change (Fig. 6).

\section{Discussion}

Exosomes derived from the endocytic pathway contain biomolecular cargo that is reflective of the physiological state of the cells secreting them [27]. Thus, exosomes provide a snapshot of a molecular signature indicative of the changes resulting from disease or therapeutic intervention. Furthermore, miRNAs transported by exosomes regulate gene expression in recipient cells [28]. Efficacy of PE in immunological, metabolic diseases, and intoxications has been attributed to the removal of humoral factors and autoantibodies [2]. Decreases in T-lymphocyte activation, B lymphocytes and proinflammatory plasma cytokines from PE has also been reported [29]. CRPS is notoriously difficult to treat $[3,4]$; however there are few reports suggesting that PE can manage symptoms, presumably by removing the patient's autoantibodies $[6,8$, $10,30]$. One study evaluating the efficacy of PE in refractory CRPS patients with a clinical presentation of a small fiber neuropathy suggested that patients with the greatest loss of small fibers and temperature sensory deficits are more likely to benefit from PE [10]. Using a multipronged approach of immune-modulating agents and

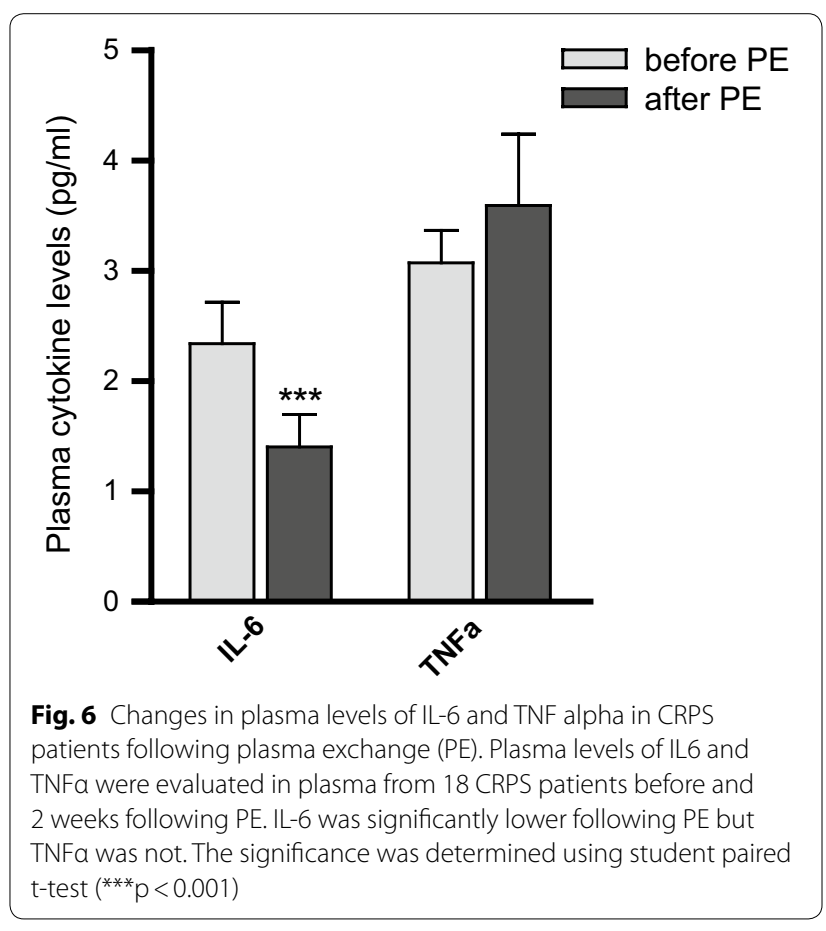

IVIG, in addition to a weekly PE regime, 91\% efficacy was observed in this previously treatment resistant patient group [10]. In these patients, pain reduction was either 
maintained with immune modulating therapies, or the pain gradually returned to pre-treatment levels. While successful in the aforementioned study, the therapeutic benefit from $\mathrm{PE}$ is variable. Moreover, there is no universally efficacious treatment for CRPS, and the mechanism by which pain evolves in CRPS or how treatments such as PE reduce pain in CRPS are unknown. All these factors necessitate the identification of a prognostic biomarker or a molecular signature to predict therapeutic efficacy of PE. We investigated the feasibility of studying exosomal miRNAs in serum of CRPS patients before and after $\mathrm{PE}$ and the regulatory role of one of the differentially expressed circulating miRNAs.

The purity and integrity of exosomes isolated conform to the guidelines put forth by the International Society for Extracellular Vesicles [21, 31] and the EV-METRIC score reported is based on the experimental parameters used. Differential expression of several miRNAs was observed in exosomes derived from responders and non-responders to PE. Since this was a retrospective study and the therapeutic outcome is known, we asked if the exosomal miRNA signature differed even prior to PE by comparing the pretreatment groups of responders and non-responders. Our analysis showed differential expression of nine miRNAs between responders and non-responders prior to treatment, suggesting two heterogeneous group of patients. This was further affirmed by the observation that PE-induced miRNA changes also differed between responders and non-responders when pre and post PE samples were compared. Both the above mentioned observations are consistent with a previous study investigating circulating miRNAs in whole blood of CRPS patients receiving ketamine therapy [32].

Amongst the differentially expressed miRNAs, miR338-5p showed a downregulation in poor responders compared to responders. Though the mechanisms underlying the development of pain are not well understood, inflammation is known to play a crucial role in CRPS. CRPS patients have significantly increased proinflammatory cytokines compared to controls [26]. Based on the bioinformatics prediction that miR338-5p can bind the $3^{\prime}$ UTR of Il6 mRNA, we sought to confirm the interaction between them. The seed sequence of miR-338-5p is ACAAUAU. In vitro validation studies confirmed the interaction between miR338-5p and IL-6. IL-6 levels in plasma was higher in responders before $\mathrm{PE}$ and considerably decreased following PE. Thus, removal of IL-6 due to PE can contribute directly to reduction in inflammation. Our studies separating responders and poor responders suggest that higher IL-6 levels (before PE) in responders are linked to miR-338-5p levels. In other words, higher IL-6 levels in responders may mediate intercellular signaling via exosomal miR-338-5p to resolve inflammation, and when the proinflammatory IL-6 is removed from circulation by PE, exosomal miR-338-5p is also significantly lowered (Table 2, Additional file 2: Table S2). Poor responders did not exhibit a notable reduction in plasma IL- 6 following PE, and hence there was no significant shift in the exosomal miR-338-5p levels. Taken together, these data suggest that determining the expression levels of miRNAs transported by exosomes in combination with cytokine analysis may be a feasible approach for patient stratification and may help predict therapeutic efficacy of PE.

The limitation of our study is the small number of patients $(n=6)$, and its retrospective nature including non-randomization. Another aspect to consider are the comorbidities associated with CRPS, how they contribute to and impact inflammation and pain. We have previously observed that CRPS patients responding poorly to ketamine had a lower body mass index (BMI) relative to responders [33]. Here we observed that all three responders have high $\mathrm{BMI}$, and two out of three poor responders have lower BMI. Additional studies representing a larger patient cohort and analysis of an array of immune markers are warranted to further investigate these preliminary observations.

\section{Conclusions}

This study provides the first evidence of differential regulation of exosomal miRNAs in CRPS patients undergoing PE. Our proof-of-concept study indicates that investigating exosomal miRNA before and after PE is a feasible approach that can be employed in the identification of a molecular signature for potential prediction of treatment response. Systems biology approaches integrating various omics data is required for identifying definitive molecular patterns associated with the disease. These studies performed on large number of samples available through biobanks from multiple sites will be needed to determine both markers associated with the diseases and for elucidation of biological pathways and processes altered under disease states [34].

\section{Additional files}

Additional file 1: Table S1. Raw data for expression of serum exosomal miRNAs in CRPS patients. Raw data and relative gene expression analysis following Taqman low density array for the estimation of 754 miRNAs in the serum exosomes of CRPS patients before and after PE. 
Additional file 2: Table S2. Plasma levels of cytokines in CRPS patient following plasma exchange (PE). Plasma levels of Fractalkine (0.33), IFNy (0.47), IL-10 (0.51), MIP-3a (0.79), IL-17A (0.31), IL-1 3, IL-2 (0.18), IL-4 (1.07), IL-6 (0.11), IL-8 (0.12),MIP-1 $\beta(0.69)$, TNFa (0.16), IL-13 (0.24), and IL-23 (3.06) were determined using MILLIPLEX MAP Human High Sensitivity T Cell Panel. Included in brackets next to each analyte is the sensitivity in $\mathrm{pg} / \mathrm{mL}$ of the assay used to determine its plasma level.

\section{Abbreviations}

ANOVA: analysis of variance; BMI: body mass index; CRPS: complex regional pain syndrome; CT: cycle threshold; IL-6: interleukin 6; IVIG: intravenous immunoglobulin; LPS: lipopolysaccharide; Na: non-responders after PE; Nb: non-responders before PE; NRS: numerical rating scale; PE: plasma exchange; Ra: responders after PE; Rb: responders before PE; TEM: transmission electron microscope; UTR: untranslated region.

\section{Authors' contributions}

SR isolated and characterized the exosomes, performed the molecular biology studies, analyzed the data, performed the statistical analysis and drafted the manuscript. SRD performed exosomal miRNA profiling. GMA measured cytokine levels and performed the statistical analysis, BS contributed to the molecular biology studies, JB participated in study design, EA diagnosed and treated the patients, AS performed miRNA analysis and the statistical analysis. SKA conceived of the study, participated in its design and coordination, analyzed data, and wrote the manuscript. All authors read and approved the final manuscript.

\section{Author details}

${ }^{1}$ Pharmacology \& Physiology, Drexel University College of Medicine, 245 North 15th Street, Mail Stop 488, Philadelphia, PA 19102, USA. ${ }^{2}$ Neurology, Drexel University College of Medicine, 245 North 15th Street, Philadelphia, PA 19102, USA. ${ }^{3}$ School of Biomedical Engineering, Science \& Health Systems, Drexel University, 3141 Chestnut Street, Philadelphia, PA 19104, USA. ${ }^{4}$ Present Address: Vincera Institute, Philadelphia, PA 19112, USA.

\section{Acknowledgements}

Not applicable.

\section{Competing interests}

The authors declare that they have no competing interests.

\section{Availability of data and materials}

The miRNA profiling data generated and analyzed for this study is included in Additional file 1: Table S1.

\section{Consent for publication}

Not applicable.

\section{Ethics approval and consent to participate}

This study was performed upon approval by Drexel University Institutional Review Board. This is a retrospective case series study of CRPS patients seen at Hahnemann University Hospital, Philadelphia. All patients met the Budapest consensus criteria for CRPS and received PE as treatment for their illness between September 2012 and June 2014. PE was performed in patients with refractory CRPS over 2 weeks. Patients with autoimmune or immunodeficiency conditions were excluded. All patients had full cardiac and neuropsychological clearance before PE. All participants agreed to provide blood samples before and after PE for this study after giving informed consent as approved by the Drexel University institutional Review Board. Patients were asked to rate their overall pain before and after the apheresis using a 11-point numerical rating scale (NRS) from 0 (no pain) to 10 (the worst pain imaginable).

\section{Funding}

This research was supported by Drexel University Clinical and Translational Research Institute to Seena Ajit and Enrique Aradillas and NIH NINDS 5R01NS102836-02 to Seena Ajit. The funders had no role in the study design, data collection and analysis, decision to publish, or preparation of the manuscript.

\section{Publisher's Note}

Springer Nature remains neutral with regard to jurisdictional claims in published maps and institutional affiliations.

Received: 31 October 2018 Accepted: 6 March 2019

Published online: 14 March 2019

\section{References}

1. Kaplan AA. Therapeutic plasma exchange: core curriculum. Am J Kidney Dis. 2008;52:1180-96.

2. Bambauer R, Latza R, Burgard D, Schiel R. Therapeutic apheresis in immunologic renal and neurological diseases. Ther Apher Dial. 2017;21:6-21.

3. Birklein F, Schlereth T. Complex regional pain syndrome-significant progress in understanding. Pain. 2015;156(Suppl 1):S94-103.

4. Bruehl S. Complex regional pain syndrome. BMJ 2015;351:h2730.

5. Kohr D, Singh P, Tschernatsch M, Kaps M, Pouokam E, Diener M, Kummer W, Birklein F, Vincent A, Goebel A, et al. Autoimmunity against the beta2 adrenergic receptor and muscarinic-2 receptor in complex regional pain syndrome. Pain. 2011;152:2690-700.

6. Hendrickson JE, Hendrickson ET, Gehrie EA, Sidhu D, Wallukat G, Schimke I, Tormey CA. Complex regional pain syndrome and dysautonomia in a 14-year-old girl responsive to therapeutic plasma exchange. J Clin Apher. 2016;31:368-74.

7. Goebel A, Shenker N, Padfield N, Shoukrey K, McCabe C, Serpell M, Sanders M, Murphy C, Ejibe A, Milligan H, et al. Low-dose intravenous immunoglobulin treatment for complex regional pain syndrome (LIPS): study protocol for a randomized controlled trial. Trials. 2014;15:404.

8. Blaes F, Dharmalingam B, Tschernatsch M, Feustel A, Fritz T, Kohr D, Singh P, Kaps M, Szalay G. Improvement of complex regional pain syndrome after plasmapheresis. Eur J Pain. 2015;19:503-7.

9. Barnett C, Wilson G, Barth D, Katzberg HD, Bril V. Changes in quality of life scores with intravenous immunoglobulin or plasmapheresis in patients with myasthenia gravis. J Neurol Neurosurg Psychiatry. 2013;84:94-7.

10. Aradillas E, Schwartzman RJ, Grothusen JR, Goebel A, Alexander GM. Plasma exchange therapy in patients with complex regional pain syndrome. Pain Physician. 2015;18:383-94.

11. Valadi H, Ekstrom K, Bossios A, Sjostrand M, Lee JJ, Lotvall JO. Exosomemediated transfer of mRNAs and microRNAs is a novel mechanism of genetic exchange between cells. Nat Cell Biol. 2007;9:654-9.

12. Conlan RS, Pisano S, Oliveira MI, Ferrari M, Mendes Pinto I. Exosomes as reconfigurable therapeutic systems. Trends Mol Med. 2017;23:636-50.

13. Fatima F, Nawaz M. Vesiculated long non-coding RNAs: offshore packages deciphering trans-regulation between cells, cancer progression and resistance to therapies. Non-coding RNA. 2017;3:10.

14. De Guire V, Robitaille R, Tetreault N, Guerin R, Menard C, Bambace N, Sapieha P. Circulating miRNAs as sensitive and specific biomarkers for the diagnosis and monitoring of human diseases: promises and challenges. Clin Biochem. 2013:46:846-60.

15. Bartel DP. MicroRNAs: target recognition and regulatory functions. Cell. 2009;136:215-33.

16. Wilczynska A, Bushell M. The complexity of miRNA-mediated repression. Cell Death Differ. 2015:22:22-33.

17. Kusaoi M, Yamaji K, Ishibe Y, Murayama G, Nemoto T, Sekiya F, Kon T, Ogasawara M, Kempe K, Tamura N, Takasaki Y. Separation of circulating MicroRNAs using apheresis in patients with systemic lupus erythematosus. Ther Apher Dial. 2016;20:348-53.

18. McDonald MK, Tian Y, Qureshi RA, Gormley M, Ertel A, Gao R, Aradillas Lopez E, Alexander GM, Sacan A, Fortina P, Ajit SK. Functional significance of macrophage-derived exosomes in inflammation and pain. PAIN ${ }^{\circledR}$. 2014;155:1527-39.

19. Orlova IA, Alexander GM, Qureshi RA, Sacan A, Graziano A, Barrett JE, Schwartzman RJ, Ajit SK. MicroRNA modulation in complex regional pain syndrome. J Transl Med. 2011;9:195.

20. Schmittgen TD, Livak KJ. Analyzing real-time PCR data by the comparative C(T) method. Nat Protoc. 2008;3:1101-8.

21. Consortium E-T, Van Deun J, Mestdagh P, Agostinis P, Akay O, Anand S, Anckaert J, Martinez ZA, Baetens T, Beghein E, et al. EV-TRACK: transparent 
reporting and centralizing knowledge in extracellular vesicle research. Nat Methods. 2017;14:228-32.

22. Wong $N$, Wang $X$. miRDB: an online resource for microRNA target prediction and functional annotations. Nucleic Acids Res. 2015;43:D146-52.

23. Agarwal V, Bell GW, Nam JW, Bartel DP. Predicting effective microRNA target sites in mammalian mRNAs. Elife. 2015;4:e05005.

24. Karagkouni D, Paraskevopoulou MD, Chatzopoulos S, Vlachos IS, Tastsoglou S, Kanellos I, Papadimitriou D, Kavakiotis I, Maniou S, Skoufos G, et al. DIANA-TarBase v8: a decade-long collection of experimentally supported miRNA-gene interactions. Nucleic Acids Res. 2018;46:D239-45.

25. Philippe L, Alsaleh G, Suffert G, Meyer A, Georgel P, Sibilia J, Wachsmann $D$, Pfeffer S. TLR2 expression is regulated by microRNA miR-19 in rheumatoid fibroblast-like synoviocytes. J Immunol. 2012;188:454-61.

26. Parkitny L, McAuley JH, Di Pietro F, Stanton TR, O'Connell NE, Marinus J, van Hilten JJ, Moseley GL. Inflammation in complex regional pain syndrome: a systematic review and meta-analysis. Neurology. 2013;80:106-17.

27. Pitt JM, Kroemer G, Zitvogel L. Extracellular vesicles: masters of intercelIular communication and potential clinical interventions. J Clin Invest. 2016;126:1139.

28. Mittelbrunn M, Gutierrez-Vazquez C, Villarroya-Beltri C, Gonzalez S, Sanchez-Cabo F, Gonzalez MA, Bernad A, Sanchez-Madrid F. Unidirectional transfer of microRNA-loaded exosomes from $T$ cells to antigenpresenting cells. Nat Commun. 2011;2:282.
29. Sadeghi M, Daniel V, Wang H, Zeier M, Schemmer P, Mehrabi A, Lahdou I, Morath C, Opelz G. Plasmapheresis adjusts inflammatory responses in potential kidney transplant recipients. Transplantation. 2013;95:1021-9.

30. Goebel A, Jones S, Callaghan T, Oomman S, Sprotte G. Treatment of long-standing complex regional pain syndrome with therapeutic plasma exchange: a preliminary case series of patients treated in 2008-2014. Pain Med. 2014;15:2163-4.

31. Lötvall J, Hill AF, Hochberg F, Buzás El, Di Vizio D, Gardiner C, Gho YS, Kurochkin IV, Mathivanan S, Quesenberry P, et al. Minimal experimental requirements for definition of extracellular vesicles and their functions: a position statement from the International Society for Extracellular Vesicles. J Extracell vesicles. 2014. https://doi.org/10.3402/jev.v3.26913.

32. Douglas SR, Shenoda BB, Qureshi RA, Sacan A, Alexander GM, Perreault M, Barrett JE, Aradillas-Lopez E, Schwartzman RJ, Ajit SK. Analgesic response to intravenous ketamine is linked to a circulating microRNA signature in female patients with complex regional pain syndrome. J Pain. 2015;16:814-24.

33. Shenoda BB, Alexander GM, Ajit SK. Hsa-miR-34a mediated repression of corticotrophin releasing hormone receptor 1 regulates pro-opiomelanocortin expression in patients with complex regional pain syndrome. J Transl Med. 2016;14:64.

34. Hasin Y, Seldin M, Lusis A. Multi-omics approaches to disease. Genome Biol. 2017;18:83.
Ready to submit your research? Choose BMC and benefit from:

- fast, convenient online submission

- thorough peer review by experienced researchers in your field

- rapid publication on acceptance

- support for research data, including large and complex data types

- gold Open Access which fosters wider collaboration and increased citations

- maximum visibility for your research: over 100M website views per year

At BMC, research is always in progress.

Learn more biomedcentral.com/submissions 